\title{
Erstmaßnahmen und Differenzialdiagnostik oraler Blutungen
}

Tim Eschbach

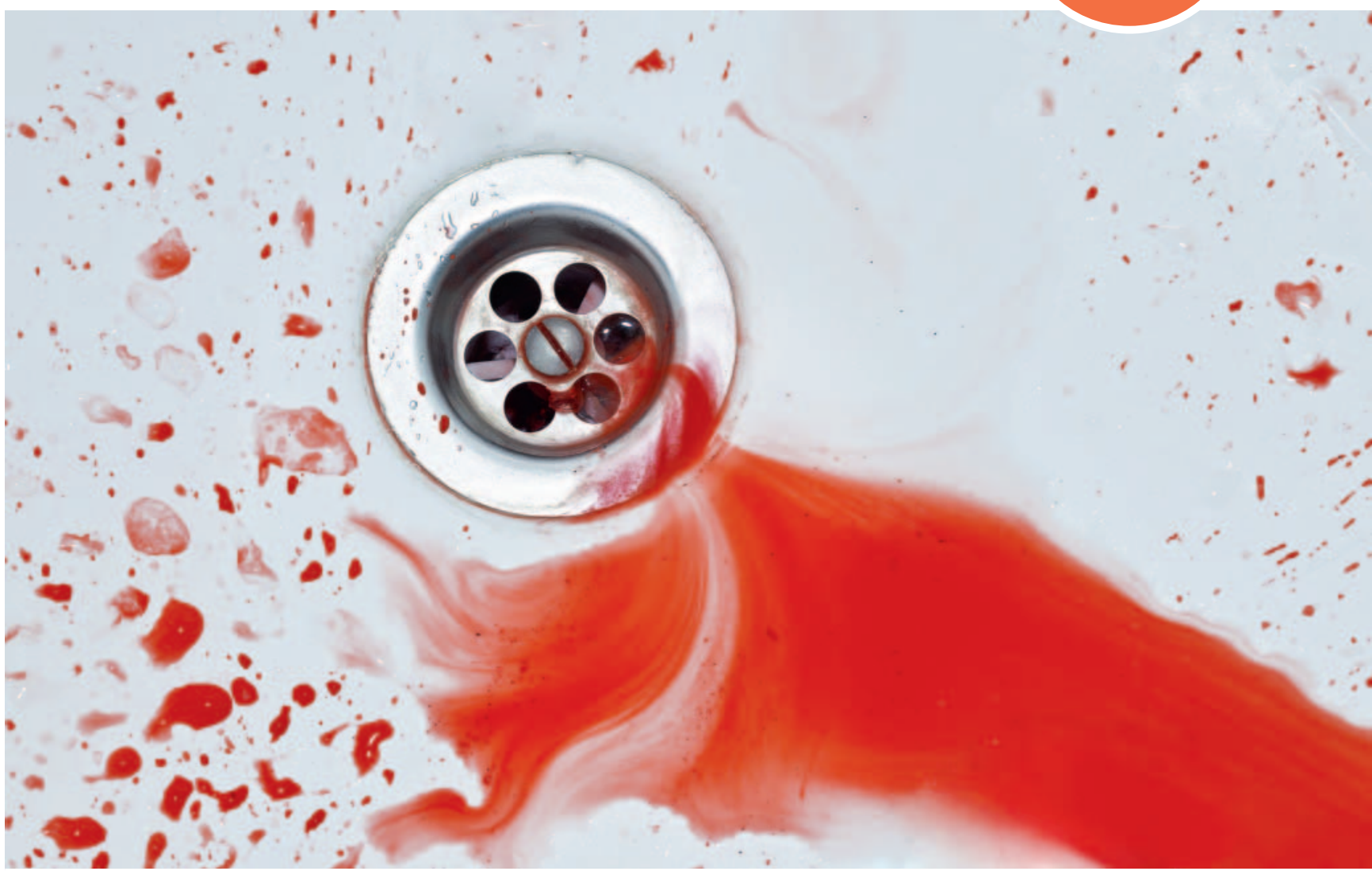

Quelle: MaksymFilipchuk/AdobeStock.com (Symbolbild)

Orale Blutungen bergen präklinisch differenzialdiagnostische Probleme. Im Vordergrund steht die Stabilisierung des Patienten. Um das geeignete Zielkrankenhaus auswählen zu können, ist eine möglichst genaue Lokalisierung der Blutungsquelle unerlässlich. Neben allgemeinen Standards der Notfallmedizin gibt es Methoden als Ultima Ratio, die eine übergangsweise Blutungskontrolle ermöglichen können.

$\begin{array}{ll}\text { ABKÜRZUNGEN } \\ \text { ABCDE } & \begin{array}{l}\text { Airway - Breathing - Circulation - } \\ \text { Disability - Exposure/Examination }\end{array} \\ \text { ASS } & \text { Azetylsalizylsäure } \\ \text { AZ } & \text { Allgemeinzustand } \\ \text { DOAK } & \text { direktes orales Antikoagulans } \\ \text { GCS } & \text { Glasgow Coma Scale } \\ \text { GI } & \text { gastrointestinal } \\ \text { Hb } & \text { Hämoglobin } \\ \text { HES } & \text { Hydroxyethylstärke }\end{array}$

HWS Halswirbelsäule

INR International Normalized Ratio

NEF Notarzteinsatzfahrzeug

P Patient

Rekap-Zeit Rekapillarisierungszeit

RR Blutdruck

RTW Rettungstransportwagen

SHT Schädel-Hirn-Trauma

syst. systolisch

VU Verkehrsunfall 


\section{FALLBEISPIEL}

Der Rettungsdienst wird von der Ehefrau des Patienten unter dem Stichwort „bewusstlose Person“ zu einem 56-jährigen Patienten gerufen. Das mitalarmierte NEF trifft kurz nach dem RTW ein. Der Patient liegt regungslos auf dem Boden im Wohnzimmer. Dort stehen leere Weinflaschen herum. Es befindet sich eine große Menge Blut um den Patienten. Der Patient ist komatös, kaltschweißig und marmoriert. Er wird folglich als kritisch eingestuft und intubiert. Unter der Verdachtsdiagnose „Ösophagusvarizenblutung“ erfolgt ein rascher Transport in die vorab informierte Klinik. Notfallmäßig wird eine Gastroskopie mit Gummibandligatur der Varizen durchgeführt. Der initiale $\mathrm{Hb}$ liegt bei $4,5 \mathrm{~g} / \mathrm{dl}$. Bei fortgeschrittener Leberzirrhose gelingt keine dauerhafte Stabilisierung. Es kommt zum Nierenversagen. Der Patient verstirbt nach 5 Tagen auf der Intensivstation.

\section{Differenzialdiagnose}

Nase, Mund- und Rachenraum sollten zunächst auf sichtbare Blutungsquellen hin inspiziert werden.

Differenzialdiagnostische Überlegungen beginnen beim nicht kritischen Patienten mit einer gezielten Anamnese, anderenfalls haben die Erstmaßnahmen nach ABCDE Priorität ( $\triangleright$ Abb. 1). Dazu sollte der Patient nach der Einnahme von Antikoagulanzien und gezielt nach relevanten Vorerkrankungen wie etwa einer vorausgegangenen UIkusblutung, Leberzirrhose (Alkoholabusus) oder Tumoren befragt werden.

Zu den relevanten Medikamenten gehören Thrombozytenaggregationshemmer wie ASS oder Clopidogrel (Plavix ${ }^{\circledR}$ ) sowie direkte orale Antikoagulanzien (DOAK) wie Rivaroxaban (Xarelto ${ }^{\circledR}$ ), Apixaban (Eliquis ${ }^{\circledR}$ ), Dabigatran (Pradaxa ${ }^{\circledR}$ ) und Edoxaban (Lixiana ${ }^{\circledR}$ ). Außerdem sollte der Patient nach der Einnahme von Phenprocoumon (Marcumar $^{\circledR}$ ) befragt werden [1]. Für einige Medikamente gibt es spezifische Antidote, sodass die Information auch für die weitere Behandlung wichtig ist.

Die genannten Medikamente führen zu einer deutlich verstärkten Blutungsneigung und -intensität. Trotz initial stabiler Verhältnisse kann sich die Blutung dramatisch entwickeln.

Merke

Blutungen bei Patienten mit Antikoagulanzieneinnahme sind daher als primär kritisch einzuschätzen.

Eine Leberzirrhose ist aufgrund der eingeschränkten Leberfunktion mit der damit einhergehenden unzureichenden Produktion von Gerinnungsfaktoren sowie durch ggf. bestehende Ösophagusvarizen ein wichtiger Risikofaktor für massive Blutungen.

Tumoren im Nasen-Rachen-Bereich oder intrapulmonal neigen ebenfalls zu Blutungen und können arterielle Gefäße arrodieren.

Neben der medizinischen Vorgeschichte wird der Patient nach Aussehen und Menge des Bluts befragt. Das wie Kaffeesatz aussehende Hämatin und schwarzer Stuhl („Teerstuhl“) deuten auf eine Blutung aus dem oberen Gastrointestinaltrakt hin. Bei ausgeprägter Blutung zeigt sich frischblutiges Erbrechen. Dies ist bei entsprechender Anamnese (Alkoholabusus?) auch Kennzeichen einer Ösophagusvarizenblutung. Blut im Sputum oder Hämoptysen sprechen eher für eine Blutung im Respirationstrakt.

Zur gezielten Therapie sollten der aufnehmenden Klinik diese Informationen unbedingt vollständig mitgeteilt werden.

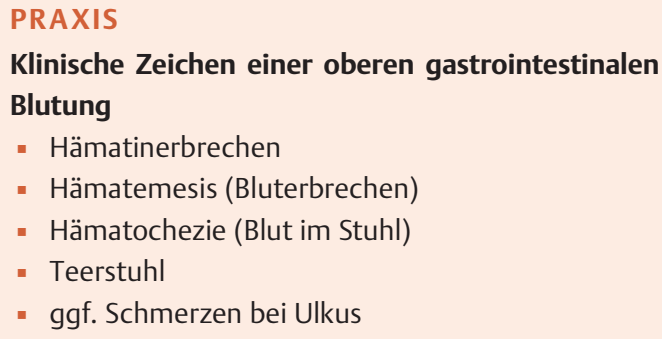

Bei traumatischen Ereignissen sind der Traumamechanismus und die Kinematik von Bedeutung, um mögliche Verletzungsfolgen abschätzen zu können. Bei einem Verkehrsunfall lohnt sich ein kurzer Blick auf das Schadensbild der beteiligten Fahrzeuge und ob ggf. Airbags ausgelöst haben. Blutungen im Mundraum können durch Verletzungen in der Nase, durch Verletzungen im Mundraum selbst oder des Gesichtsschädels entstehen. Auch ein Schädel-Hirn-Trauma (SHT) mit Schädelbasisfrakturen kann zu relevanten Blutungen im Mundraum führen.

Neben Unfallmechanismen sind auch Verletzungen im Rahmen von stumpfer oder scharfer Gewalt gegen Kopf und Hals als mögliche Ursachen denkbar. Dabei sollte der Eigenschutz nicht vernachlässigt werden. Informationen von Passanten oder der Polizei können hilfreich sein.

\section{Erstmaßnahmen}

\section{A/B-Probleme

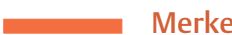

Ein Atemwegsproblem muss durch unverzügliches Absaugen von Blut und entsprechende Lagerung des Patienten beseitigt werden. 


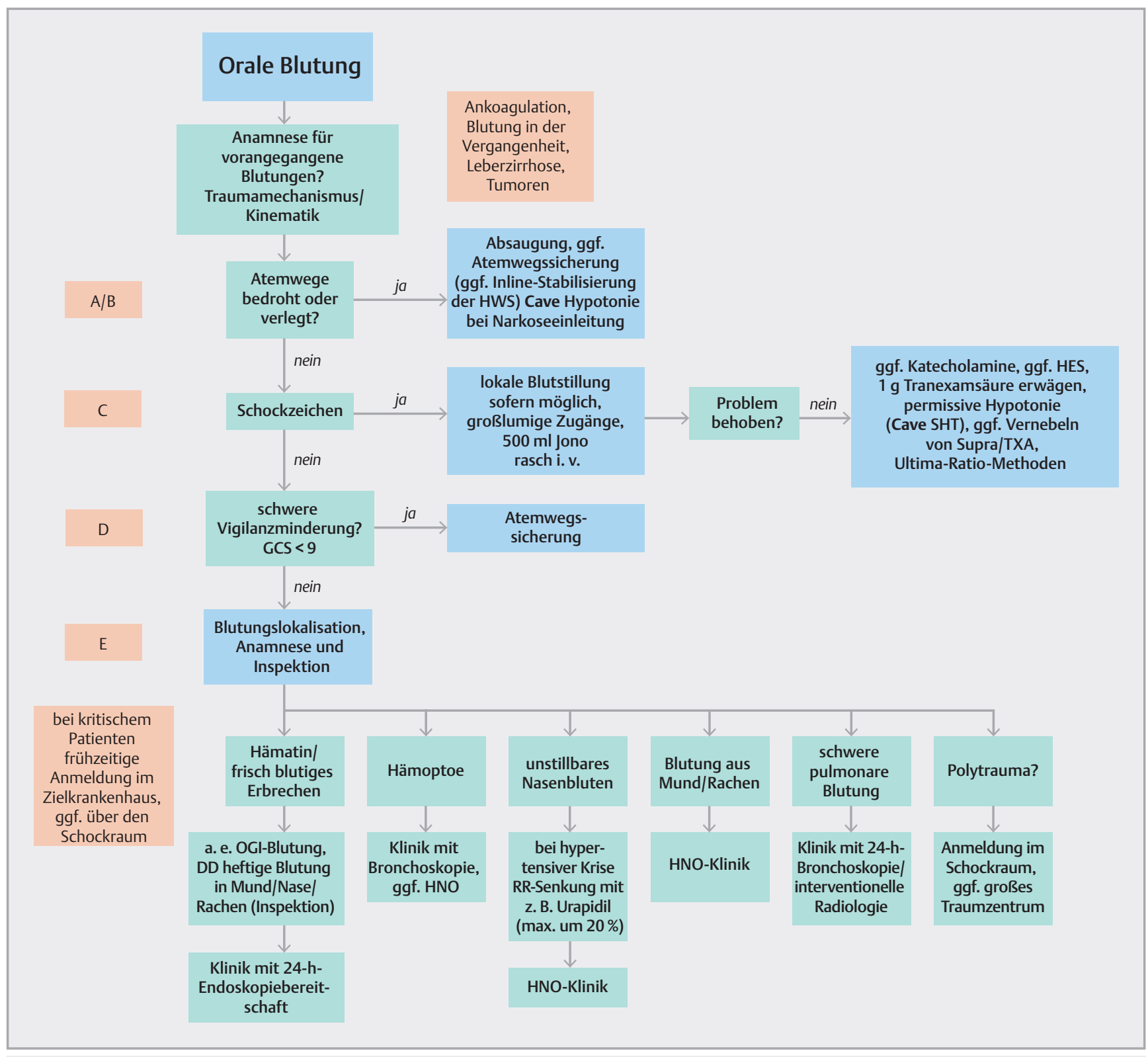

- Abb. 1 Erstmaßnahmen bei oralen Blutungen. TXA=Tranexamsäure.

Bei weiterhin unsicherem Atemweg, inadäquatem Anstieg der peripheren Sättigung oder insuffizienter Atmung müssen die Atemwege gesichert werden. Es liegt in diesem Fall ein erwartet schwieriger Atemweg vor, der durch entsprechende Vorbereitung (Plan B!) und evtl. den Einsatz eines Videolaryngoskops antizipiert werden sollte [2]. Zu beachten ist, dass bereits geringe Mengen Blut auf der Linse des Videolaryngoskops zu einem totalen Sichtverlust führen können. Dann sind Videolaryngoskope mit Macintosh-ähnlichem Spatel zu empfehlen, die eine direkte Laryngoskopie ermöglichen. Somit ist bei verdeckter Optik noch eine „klassische“ Intubation unter Sicht möglich [3]. Hat man ein Videolaryngoskop mit stark gekrümmtem Spatel, empfiehlt es sich, die speziell dafür gebogenen Führungsstäbe zu nutzen. Bei star- ken aktiven Blutungen, die die Sicht auf die Epiglottis erschweren, kann ein Absaugkatheter durch den Tubus geführt werden. Die Passage der Stimmritze erfolgt dann unter ständiger Absaugung.

\footnotetext{
Merke

Bei entsprechendem Traumamechanismus und Gefahr einer HWS-Verletzung sollte bei der Atemwegssicherung stets eine manuelle Inline-Stabilisierung der Halswirbelsäule erfolgen [3].
}

Sind die Atemwege gesichert, wird der Patient unter Einhaltung von Normokapnie, Normoxie und Normoventilation kontrolliert beatmet. 
Cave

Durch die Narkoseeinleitung besteht die Gefahr einer Hypotonie!

\section{FALLBEISPIEL}

Der Rettungsdienst wird zu einem schweren Verkehrsunfall alarmiert. Die Meldung lautet: „VU Pkw gegen Baum, P-klemmt“.

Bei Eintreffen auf einer Landstraße zeigt sich ein Pkw, der frontal gegen einen Baum geprallt ist. Aufgrund der schweren Deformation des Wagens scheint der Pkw eine hohe Aufprallgeschwindigkeit gehabt zu haben. Die Airbags haben ausgelöst. Der Fahrer ist der einzige Insasse. Er ist unter dem Armaturenbrett eigeklemmt. Auf der Stirn zeigt sich eine stark blutende Platzwunde. Es ist viel Blut im Mund zu sehen. Zunächst ist jedoch nicht klar, ob es sich um Blut von der Platzwunde handelt. Der GCS beträgt 8 .

Nach Befreiung durch die Feuerwehr zeigen sich im Primary Assessment neben dem D-Problem ein A-Problem (große Mengen Blut im Mund) sowie ein C-Problem (Instabilität beider Oberschenkel). Es erfolgen die Gabe von $1 \mathrm{~g}$ Tranexamsäure und die Intubation. Trotz sistierter Blutung am Kopf zeigt sich weiterhin ein kontinuierlicher Blutfluss aus Mund und Nase. Die Quelle lässt sich im RTW nicht lokalisieren. Nach Anmeldung als „Polytrauma“ im Schockraum des 20 min entfernten Maximalversorgers erfolgt der Transport. Dort werden eine beidseitige Oberschenkelfraktur sowie eine Fraktur der Schädelbasis nachgewiesen.

\section{C-Problem}

Die Beurteilung des Kreislaufs erfolgt über das Tasten des Radialispulses. Dabei werden die Pulsqualität und die Pulsfrequenz beurteilt sowie Hautkolorit (grau, blass, marmoriert) und die Hautqualität (z. B. kaltschweißig) geprüft. Gleichzeitig kann die Rekap-Zeit als Maß für die Mikrozirkulation getestet werden (s. Infobox). Werte über 2 Sekunden gelten als pathologisch und sind ein frühes Zeichen eines einsetzenden hämorrhagischen Schocks [4]. Der Schockindex dagegen verändert sich erst im dekompensierten Schockgeschehen und detektiert ihn somit erst (zu) spät. Eine erhöhte Herzfrequenz wird zudem z. B. durch $\beta$-Blocker maskiert.

Merke
Übersicht
Frühe Schockzeichen
- verlängerte Rekap-Zeit
- Marmorierung und Kaltschweißigkeit der Haut
- erhöhte Atemfrequenz

Sind Zeichen eines beginnenden Schocks vorhanden, sollte zumindest ein peripherer Zugang gelegt werden und es sollten rasch $500 \mathrm{ml}$ Vollelektrolytlösung infundiert werden. In kritischen Blutungssituationen und bei unzureichender Kreislaufstabilisierung nach Gabe von kristalloiden Lösungen kann Hydroxyethylstärke (HES) im Ausnahmefall eine Option sein [5]. Des Weiteren können Vasopressoren wie Cafedrin und Theodrenalin (Akrinor ${ }^{\circledR}$ ) oder Noradrenalin $\left(\right.$ Arterenol ${ }^{\circledR}$ ) bei sonst nicht zu beherrschender Kreislaufinstabilität eingesetzt werden. Es sollte dann eine permissive Hypotonie angestrebt werden (RR $90 \mathrm{mmHg}$ syst.). Eine definitive Blutungskontrolle ist i. d. R. erst im Krankenhaus möglich, sodass ein rascher Transport angestrebt werden sollte.

\section{Keine permissive Hypotonie bei Schädel-Hirn-Trauma (SHT)!}

Bei Nasenbluten im Rahmen einer hypertensiven Krise sollte der Blutdruck vorsichtig gesenkt werden [6]. Dazu kann beispielsweise Urapidil (Ebrantil ${ }^{\circledR}$ ) eingesetzt werden.

Bei kritischer, traumatischer Blutung sollte möglichst früh $1 \mathrm{~g}$ Tranexamsäure appliziert werden [7]. Eine generelle Empfehlung zur Gabe von Tranexamsäure bei atraumatischen Blutungen gibt es derzeit nicht. Die Gabe von $1 \mathrm{~g}$ Tranexamsäure i. v. kann im Einzelfall erwogen werden, um der Hyperfibrinolyse entgegenzuwirken.

Merke
Bei jeder Form der kritischen Blutung ist auf den Wärmeerhalt zu achten.

Cave

Eine Überinfusion führt zu einem Verdünnungseffekt mit erhöhter Blutungsneigung.

\section{FALLBEISPIEL}

Der Rettungsdienst wird unter dem Stichwort „AZReduktion“ ins Pflegeheim alarmiert. Bei Eintreffen findet sich ein ca. 80-jähriger Patient liegend im Bett. Auf Nachfrage berichtet die Pflegekraft, dass der Patient mehrfach erbrochen habe. Am Mund und auf dem T-Shirt sind kaffeesatzartige Reste zu erkennen. Der Patient berichtet, er habe schwarzen Stuhlgang. Im Medikamentenplan ist Marcumar aufgeführt. Der initiale Blutdruck wird mit $90 \mathrm{mmHg}$ gemessen.

Nach Legen eines großlumigen Zugangs und Infusion von $500 \mathrm{ml}$ kristalloider Lösung erfolgt der zügige Transport unter dem Verdacht auf obere GI-Blutung. In der notfallmäßig durchgeführten Gastroskopie zeigt sich ein Ulcus ventriculi mit Zeichen einer stattgehabten Blutung. Der INR ist mit 6,5 deutlich zu hoch. 


\section{D-Problem}

Ein GCS < 9 gilt als Zeichen des unsicheren Atemwegs und erfordert eine Intubation [2]. Grundsätzlich berücksichtigt werden sollten dabei

- das persönliche Können,

- die eigene Erfahrung,

- die Transportzeit zum nächsten geeigneten Krankenhaus sowie

- die Zeitverzögerung durch eine Atemwegssicherung.

Unter Umständen kann aus einsatztaktischen Gründen auf eine Schutzintubation zugunsten eines präklinisch nicht beherrschbaren C-Problems verzichtet werden.

\section{E-Problem}

Je nach vermuteter Blutungsquelle ist eine andere Zielklinik geeignet. Insbesondere dort, wo kein Maximalversorger zur Verfügung steht, ist die Auswahl der Zielklinik wichtig. Daher sollte präklinisch eine Eingrenzung der Blutungsquelle vorgenommen werden.

Für eine obere GI-Blutung wird eine 24-h-Endoskopiebereitschaft benötigt. In vielen Häusern muss diese zunächst mobilisiert werden. Unstillbare Blutungen aus der Nase müssen meist durch einen HNO-Arzt versorgt werden [6]. Bei pulmonalen Blutungen sollte eine Klinik mit 24-h-Bronchoskopiebereitschaft und ggf. interventioneller Radiologie angefahren werden. Bei schwer traumatisierten Patienten ist eine Anmeldung im Schockraum sinnvoll.

PRAXIS

Tipp

Das aufnehmende Haus sollte in jedem Fall vorab informiert werden, um Bereitschaften rechtzeitig mobilisieren zu können.

\section{FALLBEISPIEL}

Der Rettungsdienst wird zu einer 75-jährigen Patientin gerufen, die über hohen Blutdruck und starkes Nasenbluten klagt. Die Patientin ist im Primary Assessment A-D-stabil. Der initial gemessene Blutdruck ist mit 225/105 mmHg deutlich erhöht. Weitere Symptome berichtet die Patientin nicht. Die Medikamentenanamnese ergibt, dass sie seit einer Lungenembolie vor 2 Monaten einen Blutverdünner einnimmt. In der Medikamentenliste findet sich Edoxaban (Lixiana ${ }^{\circledR}$ ). Nach Gabe von fraktioniert 10 mg Urapidil (Ebranti ${ }^{\circledR}$ ) sinkt der Blutdruck auf 175/95 mmHg. Dennoch zeigt sich weiterhin ein massives Nasenbluten, sodass die Entscheidung zum Transport in die nächste HNO-Klinik gemeinsam im Team gefällt wird.

\section{Ultima-Ratio-Maßnahmen}

Bei massiver Blutung aus der Lunge kann als Ultima Ratio eine einseitige Intubation versucht werden. Dies wird präklinisch durch ein absichtlich tiefes Einführen des Endotrachealtubus aufgrund der Anatomie bis in den rechten Hauptbronchus durchgeführt. Hat der Patient eine Blutung im Bereich der linken Lunge, kann so zumindest einseitig beatmet werden und durch die Blockung des Tubus im rechten Hauptbronchus ein weiteres Eindringen von Blut in die rechte Lunge verhindert werden. Erweiterte Maßnahmen (Bronchoskopie, Doppellumentubus) sind meist nur in einer Klinik mit entsprechender Erfahrung möglich.

Bei massiver gastrointestinaler Blutung und Unmöglichkeit einer visuellen Darstellung der Stimmritzen kann eine blinde Intubation des Ösophagus mit einem Endotrachealtubus und (Über-)Blockung des Cuffs versucht werden. Die Blutung wird dabei so weit im Rachenraum komprimiert, dass unter Absaugung der Eingang der Trachea dargestellt und mit einem weiteren Tubus intubiert werden kann.

Bei der Absaugung sollte auf einen ausreichenden Durchmesser des Absaugtubus geachtet werden. Außerdem sollte bei massiver Blutung damit gerechnet werden, dass der Beutel an der Absaugpumpe rasch gefüllt sein kann und eine weitere Absaugung dann bis zum Wechsel des Beutels nicht mehr möglich ist.

PRAXIS

Tipp

Sofern verfügbar, sollten zwei Absaugpumpen bereit zum Einsatz sein (Absaugung aus dem NEF holen).

Unstillbare und bedrohliche Blutungen aus der Nase können durch Einführen eines Blasenkatheters über ein Nasenloch, Blockung des Ballons im Rachenraum und „Rückzug“ über die Nase tamponiert bzw. das massive Abfließen von Blut in den Rachenraum verhindert werden (Cave: Kontraindikation bei Schädel-Hirn- bzw. Mittelgesichtstrauma).

Die Blutstillung bei diffusen Schleimhautblutungen insbesondere im Nasen-Rachen-Raum kann durch Vernebelung von Suprarenin und/oder Tranexamsäure (Cave: OffLabel-Use) unterstützt werden.

\section{Fazit}

Präklinisch ist eine Blutungskontrolle meist nicht oder unzureichend möglich, sodass dann allgemeine notfallmedizinische Standards zur Anwendung kommen. Letztlich soll- 
te der Patient möglichst zeitnah in eine geeignete Klinik transportiert werden, um ihn rasch einer definitiven Therapie zuführen zu können. In diesem Zusammenhang ist die Vorabinformation der aufnehmenden Klinik wichtig, um etwaige Bereitschaften rechtzeitig zu mobilisieren.

Neben den allgemeinen Standards können außergewöhnliche Methoden angewendet werden, um die Zeit bis zur Klinik zu überbrücken. Diese sind als Ultima-Ratio-Methoden zu sehen und dem Einzelfall vorbehalten.

\section{KERNAUSSAGEN}

- Differenzialdiagnostische Überlegungen bei oralen Blutungen sind präklinisch eine Herausforderung.

- Die Lokalisation der Blutungsquelle ist wichtig für das Vorgehen, die Einsatztaktik und die Wahl des Zielkrankenhauses.

- Wichtig ist die Anamneseerhebung bezüglich relevanter Vorerkrankungen und der Einnahme von Antikoagulanzien. Außerdem sind der Traumamechanismus und die Beurteilung der Kinematik essenziell.

- Neben dem C-Problem durch einen hämorrhagischen Schock sind vor allem das A-Problem und die Gefahr der Aspiration bedeutsam.

- Auf frühe Zeichen eines Schockgeschehens sollte geachtet werden.

- Bei hämorrhagischem Schock kann die Gabe von Tranexamsäure erwogen werden.

- Eine Vorabinformation der Zielklinik sollte frühzeitig erfolgen, damit etwaige Bereitschaften rechtzeitig mobilisiert werden können.

- Als Ultima Ratio stehen für den Einzelfall außergewöhnliche Methoden zur Auswahl, um eine temporäre Blutungskontrolle zu erreichen.

Interessenkonflikt

\section{Erklärung zu finanziellen Interessen}

Forschungsförderung erhalten: nein; Honorar/geldwerten Vorteil für Referententätigkeit erhalten: nein; Bezahlter Berater/interner Schulungsreferent/Gehaltsempfänger: nein; Patent/Geschäftsanteile/Aktien (Autor/Partner, Ehepartner, Kinder) an Firma (Sponsor der Veranstaltung): nein; Patent/ Geschäftsanteile/Aktien (Autor/Partner, Ehepartner, Kinder) an Firma (Nicht-Sponsor der Veranstaltung): nein

Erklärung zu nichtfinanziellen Interessen

Die Autorinnen/Autoren geben an, dass kein Interessenkonflikt besteht.

\section{Autorinnen/Autoren}

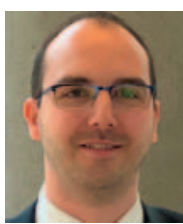

\section{Tim Eschbach}

Dr. med., Facharzt für Innere Medizin, Zusatzbezeichnung Notfallmedizin. Studium der Humanmedizin 2007-2013 an der Universität zu Köln. Tätig als leitender Arzt der Zentralen Notauf-

nahme, GFO Kliniken Rhein-Berg, Betriebsstätte Marien-Krankenhaus. Seit 2016 aktiv im

Notarztdienst, AMLS- \& PHTLS-Provider, Schwerpunkte: Notfallmedizin, Innere Medizin und Gastroenterologie.

\section{Korrespondenzadresse}

\section{Dr. med. Tim Eschbach}

GFO Kliniken Rhein-Berg, Betriebsstätte Marien-Krankenhaus Zentrale Notaufnahme

Dr.-Robert-Koch-Straße 18

51465 Bergisch Gladbach

tim.eschbach@mkh-bgl.de

\section{Zitierweise für diesen Artikel}

Dieser Beitrag ist eine aktualisierte Version des Artikels: Eschbach T. SOP Erstmaßnahmen und Differenzialdiagnostik atraumatischer oraler Blutungen. Notfallmedizin up2date 2018; 13(04): 358-360. doi:10.1055/a-0753-3396

\section{Literatur}

[1] Pohlmann A, Trebicka J, Götz M et al. Gastrointestinale Blutung. Gastroenterol Up2date 2018; 14: 43-61. doi:10.1055/s-0043121449

[2] Cavus E, Byhahn C, Meininger D et al. Management des schwierigen Atemwegs unter Extrembedingungen. Notfallmed Uup2date 2015; 10: 149-170. doi:10.1055/s-0033-1358151

[3] Timmermann A, Böttiger B, Byhahn C et al. S1-Leitlinie prähospitales Atemwegsmangement, 02/2019. Im Internet: https://www.awmf.org/leitlinien/detail/l/001-040.html (Stand: 23.10.2019)

[4] National Association of Emergency Medical Technicians (NAEMT), Hrsg. Präklinisches Traumamanagement Prehospital Trauma Life Support (PHTLS). München: Elsevier 2016: 136

[5] Werner C, Geldner G, Koch T. Hydroxyethylstärke: Stellungnahme der Präsidenten der DGAI und des BDA sowie der Präsidentin der DAAF. Anästhesiol Intensivmed Notfallmed Schmerzther 2013; 48: 437. doi:10.1055/s-0033-1352487

[6] Beck R, Sorge M, Schneider A et al. Therapiekonzepte der Epistaxis in Praxis und Klinik. Dtsch Arztebl Int 2018; 115: 12-22. doi:10.3238/arztebl.2018.0012

[7] Schweigkofler U, Trentzsch H. S3-Leitlinie Polytrauma/Schwerverletzten-Behandlung - ein Update. Notfallmed Up2date 2018; 13 (2): 171-184

\section{Bibliografie}

DOI https://doi.org/10.1055/a-0961-3039

retten 2020; 9: 49-56

(c) Georg Thieme Verlag KG, Stuttgart · New York ISSN 2193-2387 


\section{Punkte sammeln auf CMIEthieme.de}

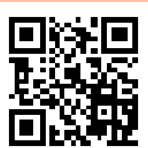

Diese Fortbildungseinheit ist in der Regel 12 Monate online für die Teilnahme verfügbar.

Den genauen Einsendeschluss finden Sie unter https://eref.thieme.de/CXDGLT].

Sollten Sie Fragen zur Online-Teilnahme haben, finden Sie unter https://cme.thieme.de/hilfe

eine ausführliche Anleitung. Wir wünschen viel Erfolg beim Beantworten

der Fragen!

Unter https://eref.thieme.de/CXDGLT] oder über den QR-Code kommen Sie direkt zur Startseite des Wissenstests.

\section{Frage 1}

Welche Aussage zur präklinischen Differenzialdiagnostik und Einsatztaktik bezüglich oraler Blutungen ist zutreffend?

A Die Lokalisation einer Blutung lässt sich präklinisch immer feststellen.

B Differenzialdiagnostisch muss an eine intrazerebrale Blutung gedacht werden.

C Die Lokalisation der Blutung spielt für die weitere Therapie und die Einsatztaktik keine Rolle.

D Bei Verdacht auf obere gastrointestinale Blutung sollte ein Krankenhaus mit 24-h-Endoskopiebereitschaft angefahren werden.

E Es sollte immer ein Krankenhaus der Maximalversorgung angefahren werden.

\section{Frage 2}

Welche anamnestischen Angaben oder Vorerkrankungen sind bei oralen Blutungen nicht von Bedeutung?
A Leberzirrhose
B Vorhofflimmern mit dauerhafter Antikoagulation
C Tumoren im Nasen-Rachen-Raum oder intrapulmonal
D Art und Menge des erbrochenen Blutes
E Hausstaubmilbenallergie

\section{Frage 3}

Welche Medikamente gehen nicht mit einem erhöhten Blutungsrisiko einher und können nicht zu einer hohen Blutungsintensität führen?
A Rivaroxaban (Xarelto ${ }^{\circledR}$ )
B Apixaban (Eliquis ${ }^{\circledR}$ )
C Clopidogrel $\left(\right.$ Plavix $\left.^{\circledR}\right)$
D Pantoprazol (Pantozol ${ }^{\circledR}$ )
E Phenprocoumon (Marcumar ${ }^{\circledR}$ )

\section{Frage 4}

Welche Aussage bezüglich einer Atemwegssicherung trifft zu, wenn orale Blutungen zu einer Gefährdung des Atemweges führen?

A Es sollte primär ein Videolaryngoskop möglichst mit Macintosh-ähnlichem Spatel verwendet werden.

B Orale Blutungen sind niemals A/B-problematisch.

C Die Vigilanz des Patienten spielt bei der Entscheidung zur definitiven Atemwegssicherung keine Rolle.

D Die Einleitung einer Narkose ist bei stark blutenden Patienten mit Schockgeschehen unproblematisch.

E Eine endotracheale Intubation ist eine einfache Prozedur, die durch jeden Rettungsdienstmitarbeiter ohne Training durchgeführt werden kann.

\section{Frage 5}

Eine schwere Blutung kann zu einem hämorrhagischen Schock führen. Welches ist kein frühes Zeichen eines Schockgeschehens?

A erhöhte Atemfrequenz

B hohe Herzfrequenz und niedriger Blutdruck

C verlängerte Rekapillarisierungszeit

D marmorierte Haut

E Kaltschweißigkeit

\section{Frage 6}

Welche Aussage zur Gabe von Tranexamsäure im Rahmen von oralen Blutungen trifft zu?

A Tranexamsäure ist aufgrund der nicht vorhandenen Nebenwirkungen unkritisch einzusetzen.

B Bei hämorrhagischem Schock kann die Gabe von Tranexamsäure erwogen werden.

C Die Gabe von Tranexamsäure ist bei oberer gastrointestinaler Blutung heute Standard.

D Tranexamsäure sollte in einer Dosis von $5 \mathrm{~g}$ intravenös verabreicht werden.

E Tranexamsäure kann ausschließlich intravenös verabreicht werden.

- Weitere Fragen auf der folgenden Seite... 


\section{Punkte sammeln auf CME.thieme.de}

\section{Frage 7}

Welche präklinisch durchführbaren Maßnahmen kommen als Ultima Ratio im Einzelfall nicht infrage?

A Bewusste einseitige Intubation zur Ventilation der nicht betroffenen Lungenseite

B Einführen eines Blasenkatheters in die Nase, Blockung im Rachen und Rückzug zur Kompression der Blutung bzw. Verhinderung des Abflusses großer Mengen Blut in den Rachen

C Bronchoskopische Blutstillung am Einsatzort

D Vernebelung von Tranexamsäure bei diffusen Schleimhautblutungen

E Bewusste Intubation des Ösophagus und Überblockung des Cuffs zur Kompression einer Blutung im Ösophagus bzw. zur Verhinderung des Rückflusses von Blut in den Rachen, anschließend endotracheale Intubation mit zweitem Tubus unter stetiger Absaugung

\section{Frage 8}

Welches Management bei hämorrhagischem Schock ist korrekt?

A Es sollten mindestens 2 I Vollelektrolytlösung infundiert werden.

B Bei nicht stillbarer Blutung sollte eine permissive Hypotonie angestrebt werden (Ausnahme: Schädel-Hirn-Trauma).

C Vasokonstriktoren wie Arterenol sind keine Option zur Anhebung des Blutdrucks bei nicht ausreichender Wirkung von kristalloiden Lösungen.

D Es sollte stets präklinisch eine Bluttransfusion erfolgen.

E Noradrenalin ist kontraindiziert bei hämorrhagischem Schock.

\section{Frage 9}

Welche Aussage hinsichtlich gastrointestinaler (GI) Blutungen ist nicht korrekt?

A Bei jeder Form der kritischen Blutung ist auf den Wärmeerhalt zu achten.

B Eine Blutung aus der Lunge sollte primär in einem Haus mit Bronchoskopiebereitschaft behandelt werden.

C Eine hypertensive Entgleisung kann eine Epistaxis auslösen.

D Bei einer oberen GI-Blutung sollte grundsätzlich sofort eine Intubation durchgeführt werden.

E Bei kritischen Patienten sollte immer eine Vorabinformation der aufnehmenden Klinik erfolgen.

\section{Frage 10}

Einer der folgenden Befunde ist kein Zeichen einer oberen gastrointestinalen Blutung. Welcher?
A Hämaturie
B Hämatinerbrechen
C Teerstuhl
D frisch blutiges Erbrechen
E ggf. Oberbauchschmerzen bei Ulcus ventriculi 